\title{
Perceiving distance: A role of effort and intent
}

\author{
Jessica K Witt, Dennis R Proffitt, William Epstein \\ University of Virginia, PO Box 400400, Charlottesville, VA 22903-2477, USA; \\ e-mail: jw2ex@cms.mail.virginia.edu \\ Received 12 May 2003, in revised form 2 February 2004
}

\begin{abstract}
Perceiving egocentric distance is not only a function of the optical variables to which it relates, but also a function of people's current physiological potential to perform intended actions. In a set of experiments, we showed that, as the effort associated with walking increases, perceived distance increases if the perceiver intends to walk the extent, but not if the perceiver intends to throw. Conversely, as the effort associated with throwing increases, perceived distance increases if people intend to throw to the target, but not if they intend to walk. Perceiving distance combines the geometry of the world with our behavior goals and the potential of our body to achieve these goals.
\end{abstract}

\section{Introduction}

The ground plane beneath our feet has two principal components: orientation and extent. The terrain's orientation, often called geographical slant, is specified relative to the gravitationally defined horizontal. The aspect of extent that is of concern in this paper is egocentric depth, which is the distance from an observer to a designated target.

Most of the research on perceiving orientation and extent has focused on the optical variables that relate to these terrain properties. Recent research, however, has suggested that there are additional factors influencing spatial perception. These studies indicate that perception of spatial layout is not only a function of optical information, but it is also a function of the perceiver's potential to act on the environment. For example, as effort for walking increases, perceived distance also increases (Proffitt et al 2003). In the current work, we refine this account by showing that perception is only influenced by the effort associated with a specific intended action. Effort for walking influences perceived distance when the perceiver intends to walk to the target, but not when the perceiver intends to throw to the target. Conversely, when effort for throwing increases, perceived distance increases when the perceiver intends to throw to the target, but not when the perceiver intends to walk to the target. We argue that perception of distance is a function of distal extent, the action that the perceiver intends to perform, and the effort associated with this action.

\subsection{The role of effort in distance perception}

In most of the research on perceiving egocentric distance, the oculomotor and optical variables associated with distal extent have been manipulated, and we have a good understanding how the information carried by these variables influences the perception of distance (Epstein and Rogers 1995; Proffitt and Caudek 2002). Few researchers have examined how non-visual factors inherent in the perceiver influence perception.

Recently, Proffitt et al (2003) showed that perception of distance is influenced by the physiological potential of the perceiver. When the anticipated effort associated with walking to a target increased, perceived distance increased. For example, apparent egocentric distance increased when participants wore a heavy backpack. This finding was preceded by several studies demonstrating that the perception of geographical slant is affected by such variables as carrying a heavy load, fatigue after a long run, level of physical fitness, age, and health (Bhalla and Proffitt 1999; Proffitt et al 1995). 
Anticipated effort relates energy expenditure to an expected perceptual outcome. For example, people anticipate that exerting some quantity of walking effort will propel them forward at a certain perceived speed. Moreover, when people increase their walking effort, by increasing the pace and length of their steps, they anticipate an increase in their apparent speed. This relationship between energy expenditure and perceptual outcomes is a learned relationship that can be adapted. Rieser et al (1995) showed that changing the relationship between walking effort and concurrent optic flow produced a visuomotor aftereffect. They had participants walk on a treadmill that was on a trailer being pulled by a tractor. The treadmill could be set to a different speed than the speed of the tractor, thereby decoupling the perceived speed from the expected speed based on expended walking energy. This mismatch between optic flow rate and walking speed evoked an aftereffect in blindwalking. In blindwalking, participants view a target, are then blindfolded, and attempt to walk to the target without vision. When the rate of optic flow was faster than the rate which normally accompanies the walking rate, blindfolded participants undershot the targets. Conversely, when the rate of optic flow was slower, blindfolded participants overshot the targets.

In related research, the same aftereffect was found when walking was paired with zero optic flow. After running on a treadmill while blindfolded, participants drifted forward when attempting to jog in place with eyes closed (Anstis 1995). In an experiment by Proffitt et al (2003), participants wore a virtual-reality head-mounted display while walking on a treadmill. For half the participants the virtual world was stationary resulting in zero optic flow, while the other half perceived a virtual world moving appropriately at the same rate as they were walking. Blindfolded participants in the zero-optic-flow condition drifted forward when attempting to walk in place, whereas those in the canonical-optic-flow condition walked forward by a significantly reduced degree. Durgin and Pelah (1999) also showed that participants drift forward when walking in place after running behind a golf cart while wearing a blindfold. In the extreme case of walking on a treadmill with zero optic flow, the recalibration causes participants to expend forward walking energy in an attempt to remain stationary while blindwalking in place. These studies demonstrate that a recalibration between walking energy and anticipated optic flow changes the anticipated effort required to walk a given extent.

In addition to behavioral aftereffects such as changes in blindwalking and walking in place, recalibration also results in perceptual aftereffects. A mismatch between energy expenditure and perceptual outcome has been shown to affect conscious distance perception (Proffitt et al 2003). Participants made verbal distance estimates to a target both before and after walking on a treadmill. While walking on the treadmill, half the participants viewed a stationary virtual world, whereas the other half viewed a virtual world moving by them at the normally accompanying rate. Those that experienced zero optic flow estimated distances to be farther away after the adaptation compared to those that experienced canonical optic flow. The participants in the zero-optic-flow condition recalibrated to the new relationship between energy expenditure and perceptual outcome. In essence, their perceptuomotor system learned that it took some forward walking energy to go nowhere, and thus that it would take more walking effort to walk a prescribed distance. These studies demonstrated that distance is perceived as a function of both distal extent and the anticipated effort required to walk the extent.

\subsection{Is the effect of effort general or functionally specific?}

The current studies were designed to investigate whether the effects of effort on perception are pervasive or action-specific. More specifically, we sought to determine whether the effects of effort on perceived distance are unconditional or dependent upon the specific behavior that participants anticipate performing. 
Research on visuomotor adaptations has shown that recalibrating the relationship between effort and a perceptual outcome causes functionally specific visuomotor aftereffects (Rieser et al 1995). When the adaptation involved a mismatch between walking energy and perceptual outcome, aftereffects were observed for blindwalking but not for throwing to targets while blindfolded or for turning in place while blindfolded. When the adaptation involved a mismatch between throwing energy and perceptual outcome, aftereffects were observed for blind throwing but not for blindwalking. Similarly, after adapting to turning in place, turning in place while blindfolded was affected whereas blindwalking was not.

The current experiments were designed to assess whether the influence of effort on distance perception is general or functionally specific. We know that increasing the effort associated with walking to a target causes that target to appear farther away (Proffitt et al 2003). We do not know whether this change in apparent distance would also occur if participants viewed the target with some other behavior in mind, such as throwing a ball to its location. In our experiment 5 , participants walked on a treadmill and experienced no optic flow. They were then asked to make distance judgments in two conditions. One group made their judgments with the expectation that they would then proceed to blindwalk to the target. The other group provided judgments with the expectation that they would next throw a beanbag to the target location. The experimental question was whether a recalibration of effort for walking would affect perceived distance if the participants were intending to throw. If the influence of effort on perception were unconditional, then we would expect perceived distance to increase regardless of the perceiver's intentions. However, if the effect of effort on perception is functionally specific, then an increase in effort for walking should only influence perceived distance when the perceiver is intending to walk. On applying this functional-specificity account to the current studies, perceived distance should increase after walking on the treadmill if participants were intending to walk but not if they were intending to throw. Similarly, if anticipated effort for throwing increased, then perceived distance should increase only when participants were intending to throw and not when they were intending to walk. According to this account, perception is a function of (i) distal layout, (ii) what the perceiver is intending to do, and (iii) the effort associated with the intended action.

\subsection{Overview of the studies}

In the first three experiments, we manipulated effort for throwing by having half of our participants throw a heavy ball and the other half throw a light ball. We found that effort for throwing influenced verbal reports of distance and a perceptual distancematching task; however, effort for throwing did not influence blindwalking. In the fourth experiment, we manipulated effort for throwing and intention for action. All participants threw a heavy ball and then made verbal estimates of distance. After making a verbal distance judgment, half of the participants blindwalked to the target while the other half threw to it blindfolded. We found that effort for throwing influenced conscious perception of distance only when the participants were preparing to throw to the target but not when they intended to walk to it. In the fifth and final experiment, we manipulated effort for walking by having people walk on a treadmill with zero optic flow. Participants made a verbal judgment of distance before and after the manipulation. After making each estimate, half the participants blindwalked to the target and half threw to the target. Thus, when making the distance judgment, half of the participants were anticipating that they would next throw to the target, whereas half were expecting to blindwalk to it. The treadmill-walking manipulation caused an increase in apparent distance judgments only for those participants who were intending to blindwalk. 


\section{Experiment 1. Throwing effort and perceived distance: verbal estimates}

Proffitt et al (2003) found that increasing anticipated walking effort led to increases in estimated distance. In this experiment, we demonstrated that effort for throwing also influences distance perception. Participants threw either a heavy ball or a light ball at targets placed in a field. Those who threw the heavy ball judged the targets to be farther away than those who threw the light one.

\subsection{Method}

2.1.1 Participants. Twenty-four University of Virginia students (twelve male, twelve female) participated. Participants were either paid or recruited as part of a requirement for an introductory psychology course. All had normal or corrected-to-normal vision. They were naïve to the purpose of the experiment and had not participated in prior distance-perception experiments.

2.1.2 Apparatus and stimuli. Participants made distance judgments in a flat, grassy field at the University of Virginia. Golf tees were used to mark distances ranging from 3 to $11 \mathrm{~m}$ from the observer. The tees were placed flush with the ground so that participants could not see them. Six rows of tees were arranged in a radial pattern with the observer being located at the center. The tees facilitated the placement of a small orange disc cone used to mark each target distance. Practice distances were placed at 3, 5, 9, and $11 \mathrm{~m}$. Test targets were placed at 4, 6, 8, and $10 \mathrm{~m}$.

Two balls were used. The light ball was a miniature basketball (diameter $=0.18 \mathrm{~m}$ ) weighing $0.32 \mathrm{~kg}$. The heavy ball was a slightly bigger medicine ball (diameter $=0.19 \mathrm{~m}$ ) that weighed $0.91 \mathrm{~kg}$. During testing, half the participants threw the light ball while the other half threw the heavy ball.

2.1.3 Design. Participants were assigned in alternating order to either the heavy-ball or the light-ball condition. Six males and six females participated in each condition. Each participant made 12 distance estimates (4 practice trials and 2 blocks of 4 test trials). Distance presentation order within the practice and test blocks was randomized. The row on which the target cone was presented was also randomized to minimize the use of environmental features as cues to distance from trial to trial.

2.1.4 Procedure. Participants threw either the heavy ball or the light ball for all of the trials. They completed one practice block and two test blocks. Each block consisted of throwing to 4 targets. The targets were placed at 3,5,9, and $11 \mathrm{~m}$ in the practice block. In each test block, targets were placed at 4, 6, 8, and $10 \mathrm{~m}$. The order of distances was randomized in each block. For each distance, participants threw the ball three times to the target. Participants were instructed to throw underhand with their dominant hand. Throwing distance was measured to the nearest $0.5 \mathrm{~m}$ by using the marks on the ground. After the third throw, participants were asked to estimate the distance to the target in feet. While the next target was being set up, participants were asked to turn around. All participants stood at the convergence point of the six radii. Viewing duration was not limited. After 4 trials, the participants were told that practice was over. Practice was used to ensure that the participants began to settle on a consistent strategy for estimating distance prior to the test trials. 8 test trials were presented.

\subsection{Results and discussion}

To assess the effect of effort for throwing on distance perception, we ran a 2 (gender) $\times 2$ (ball) $\times 2$ (test block) $\times 4$ (distance) ANOVA with repeated measures on the last two factors. The dependent factor was participants' verbal estimates.

Results showed a main effect for ball $\left(F_{1,20}=6.147, p<0.05\right)$. Participants who threw the heavy ball estimated distances to be farther than participants who threw the 


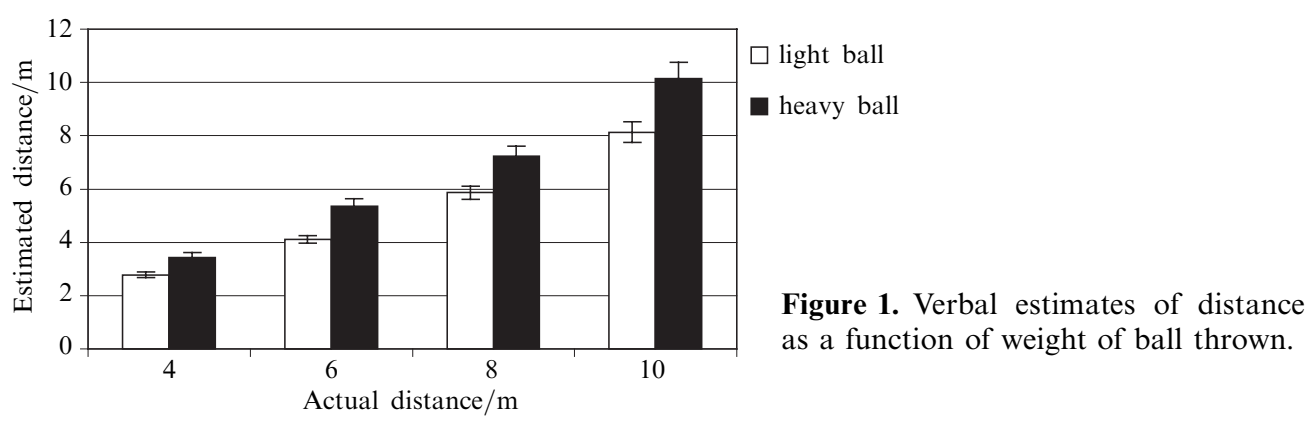

light ball (see figure 1). This is consistent with our hypothesis that effort for throwing influences conscious distance perception.

There was a significant main effect for distance $\left(F_{3,60}=254.141, p<0.001\right)$. No other effects were significant.

There was no difference between the two groups in how far they actually threw the balls (light ball: mean $=6.79 \mathrm{~m}, \mathrm{SD}=0.32 \mathrm{~m}$; heavy ball: mean $=6.58 \mathrm{~m}, \mathrm{SD}=0.13 \mathrm{~m}$ ). We further analyzed the relationship between actual throwing performance and verbal estimates. For each trial, we computed throwing error by subtracting the actual distance from the mean thrown distance and verbal estimate error by subtracting the actual distance from the verbal estimate. We found no correlation between these two measures $(r=-0.029, p>0.6)$. These analyses indicate that perceived distance was unrelated to actual throwing performance. For this reason, actual throwing distance was not assessed in subsequent experiments.

Although it appears that participants who threw the heavy ball were more accurate in their judgments of distance, most research on distance perception shows that distances in this range are generally underestimated (Amorim et al 1998; Loomis et al 1992; Norman et al 1996). The important conclusion that we draw from this experiment is that participants in the heavy-ball condition perceived the targets as being farther away than did those who threw the lighter ball.

\section{Experiment 2. Throwing effort and perceived distance: perceptual matching}

Experiment 1 demonstrated that effort for throwing influences verbal reports of distance. In experiment 2 we showed that the same throwing manipulation influences another measure of conscious distance perception. Participants were asked to match the egocentric distance to the target by adjusting the separation between two poles placed in the frontoparallel plane near the target. Two experimenters holding the poles moved closer or farther apart according to the participants' instructions until the participants judged the distance between the two poles to be the same as their distance to the target. Participants who threw the heavy ball made the separation between the two poles greater than did those who threw the lighter ball.

\subsection{Method}

3.1.1 Participants. Ninety-six University of Virginia students (forty-eight male, forty-eight female) participated. Participants were either paid or recruited as part of a requirement for an introductory psychology course. All had normal or corrected-to-normal vision. They were naïve to the purpose of the experiment and had not participated in prior distance-perception experiments.

3.1.2 Apparatus and stimuli. The experiment was run on a flat, grassy field at the University of Virginia. Spray paint was used to mark target locations. The grass was thick enough that the spray paint was not visible from the participants' perspective. Targets were placed along one of six radii. Two practice targets were placed at 5 and 
$9 \mathrm{~m}$ and 4 test targets were placed at 4, 6, 8, and $10 \mathrm{~m}$. A small orange disc cone was used to mark each target distance.

The same two balls as in the first experiment were used. Two white poles $1.5 \mathrm{~m}$ long and $0.02 \mathrm{~m}$ wide were used for matching the distances. Marks were placed at twice the target distance along a line intersecting the target and perpendicular to the line of sight. These were used to determine the initial placement of the poles in the 'out' condition (see figure 2).

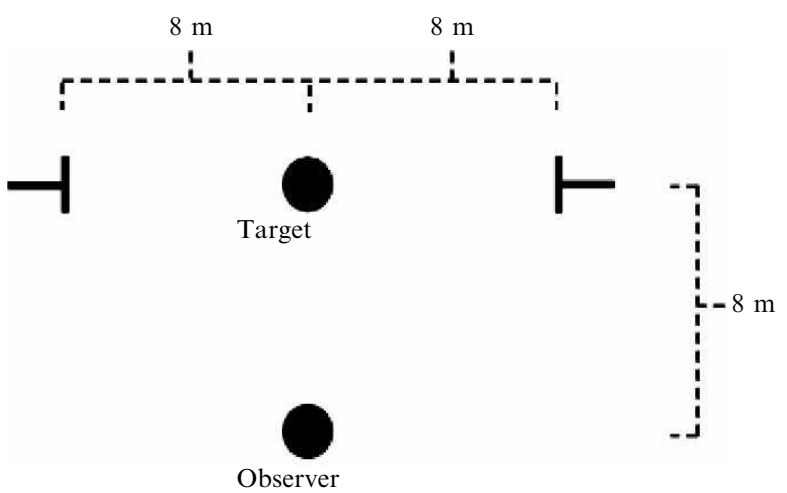

Figure 2. A birds-eye view of the target space with an example target at $8 \mathrm{~m}$. Stimuli were positioned 4 to $10 \mathrm{~m}$ from the observer along one axis. Marks were placed at twice the distance to the target in the horizontal plane. Poles either started at the marks on the outside and moved towards the target ('out' condition), or they started at the target and moved towards the marks on the outside ('in' condition).

3.1.3 Design. Participants were assigned to either the heavy-ball or the light-ball condition. They were also assigned to one of two pole conditions: 'in' or 'out'. In the 'in' condition, the poles were initially placed directly behind the target and moved towards the doubled marks. In the 'out' condition, the poles were initially placed on the doubled marks and moved towards the target. Twelve male and twelve female participants were in each of the four conditions. Each participant made 6 distance estimates ( 2 practice trials and 4 test trials). Their presentation order was randomized.

3.1.4 Procedure. Participants threw either the heavy ball or the light ball for all of the trials. They completed 2 practice distances and 4 test distances. The practice targets were placed at 5 and $9 \mathrm{~m}$ and the test targets were placed at 4, 6, 8, and $10 \mathrm{~m}$. Practice distances were alternated and test distances were randomized.

For each distance, participants threw the ball three times to each target. Participants were instructed to throw underhand with their dominant hand. After the third throw, two experimenters held up the two poles. In the 'in' condition, the poles were held up directly behind the target. In the 'out' condition, the poles were held up at the doubled marks. While holding the ball, participants gave instructions to the experimenters about whether to move towards or away from each other until they determined that the distance between the two poles matched the egocentric distance to the target. Then they were asked to make a final throw to the target. While the next target was being set up, participants were asked to turn around.

\subsection{Results and discussion}

To determine whether effort for throwing affected the visual distance-matching task, we ran a $2 \times 2 \times 2 \times 4$ ANOVA with repeated measures on the last factor. The independent factors were gender, ball, pole condition, and distance. The dependent factor was distance between the poles measured in meters after the participant had matched the distance between the poles to the distance to the target.

The main effect for ball was significant $\left(F_{1,88}=7.272, p<0.01\right)$. Participants who threw the heavy ball positioned the poles to be farther apart than participants who threw the light ball (see figure 3 ). The main effect for pole condition was significant $\left(F_{1,88}=46.173, p<0.001\right)$. Participants in the 'in' condition positioned the poles 


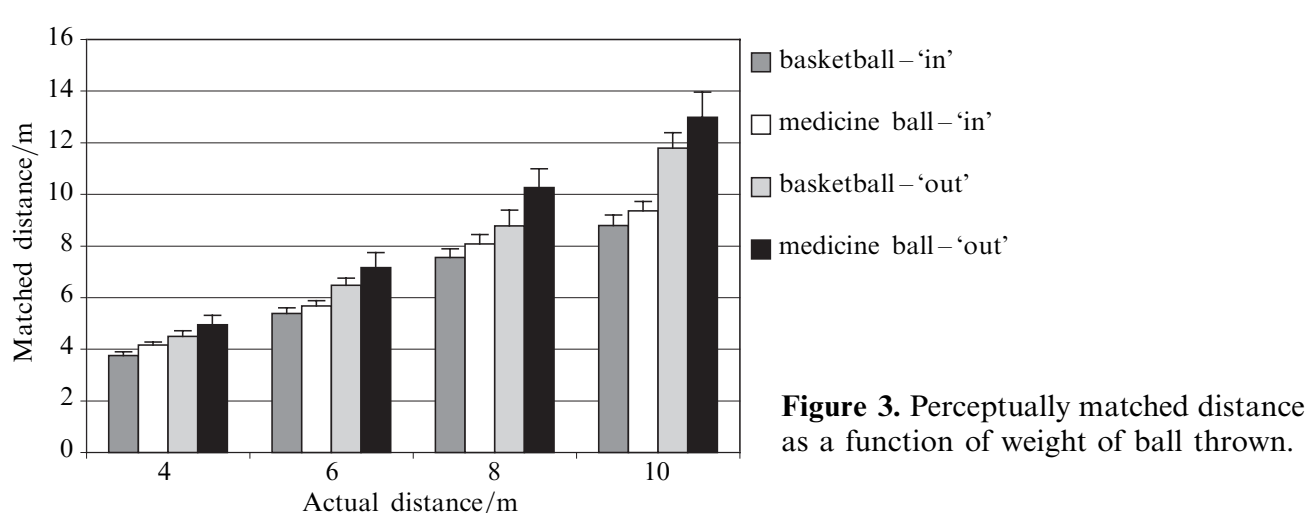

to be closer than participants in the 'out' condition. The main effect for distance was significant $\left(F_{3,264}=915.169, p<0.001\right)$. The main effect for gender was not significant. The only interaction that was significant was the interaction between pole condition and distance $\left(F_{3,264}=41.365, p<0.001\right)$. These results show that effort for throwing influences apparent egocentric distance judgments.

\section{Experiment 3. Throwing effort and perceived distance: blindwalking}

Experiments 1 and 2 showed that effort for throwing influences conscious perception of distance. However, the throwing manipulation used by Rieser et al (1995) was found not to influence blindwalking. In this experiment, as in the previous two, participants threw either the heavy or the light ball. After throwing, they blindwalked to the targets. Consistent with Rieser et al, we found that manipulating effort for throwing did not influence blindwalking, even though in the previous two experiments it was found that this same manipulation did influence apparent distance judgments as assessed via verbal reports and a perceptual-matching task.

\subsection{Method}

4.1.1 Participants. Twenty University of Virginia students (ten male, ten female) participated. Participants were either paid or recruited as part of a requirement for an introductory psychology course. All had normal or corrected-to-normal vision. They were naïve to the purpose of the experiment and had not participated in prior distanceperception experiments.

4.1.2 Apparatus and stimuli. The same setup and balls as in experiments 1 and 2 were used. In addition, a small blindfold was employed.

4.1.3 Design. Participants were assigned to either the heavy-ball or the light-ball condition. Five male and five female participants were in each condition. Each participant blindwalked to the 6 target distances (2 practice trials and 4 test trials). Their presentation order was randomized.

4.1.4 Procedure. Participants threw either the heavy ball or the light ball for all of the trials. They completed 2 practice distances and 4 test distances. The practice targets were placed at 5 and $9 \mathrm{~m}$, and the test targets were placed at $4,6,8$, and $10 \mathrm{~m}$. Practice distances were alternated and test distances were randomized.

For each distance, the participants threw the ball to each target until they hit it, or no more than three times. Participants were instructed to throw underhand with their dominant hand. After the last throw, the participant put on the blindfold and was turned more than $180^{\circ}$ to face a new direction. This was to ensure that participants were responding to the distance to the target and not to the location of the target. 
Then the participant attempted to walk the perceived target distance. The distance that the participant walked was measured in meters, and an experimenter led the participant in a rounded path back to the starting point. The target was placed at the next test distance before the subject was allowed to remove the blindfold.

\subsection{Results and discussion}

A $2 \times 2 \times 4$ ANOVA with repeated measures on the last factor was run in order to determine whether effort for throwing influenced blindwalking. The independent factors were gender, ball, and distance. The dependent factor was distance walked blindfolded. The main effect for distance $\left(F_{3,48}=148.587, p<0.001\right)$ was the only significant effect. These results suggest that the effect of effort for throwing does not extend to blindwalking (see figure 4 ).

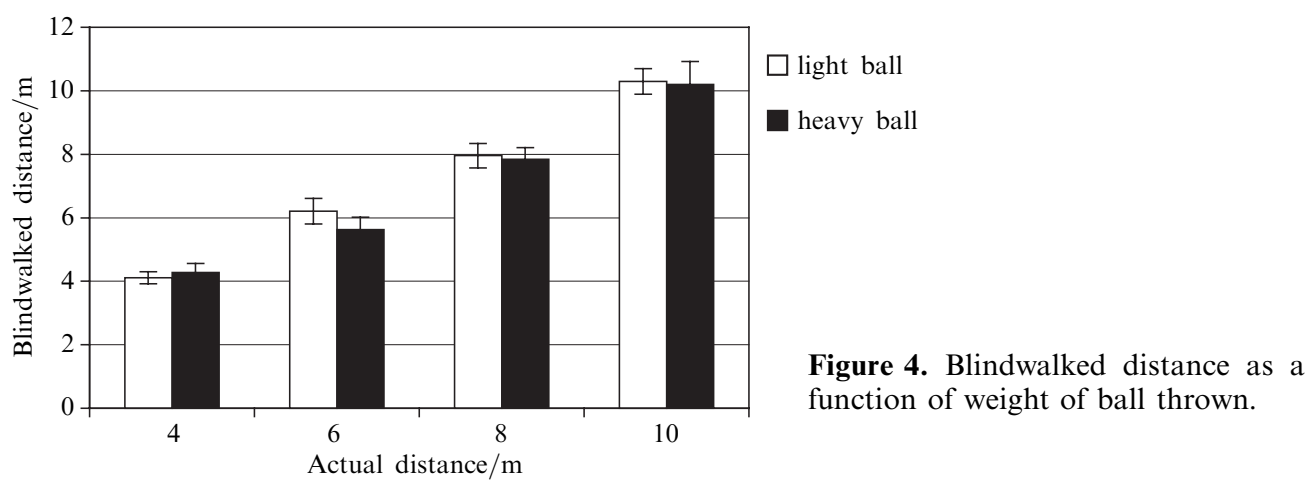

\section{Experiment 4. Perceived distance: effort for throwing and intention}

In experiment 1, participants completed a sequence of throws to targets and then made distance estimates. This sequence was repeated such that, after each estimate, participants expected to throw again. Those who threw the heavy ball reported targets to be farther away than those who threw the light ball. In the following experiment, all participants threw the heavy ball and it was their intentions that were manipulated. The intention manipulation was defined by the action that the participants were anticipating performing immediately after making each distance judgment. Participants in one group knew that, after making each distance estimate, they would blind throw to the target, whereas those in the other group knew that they would blindwalk to it. Thus, at the time that they were making distance estimates, half of the participants were anticipating that they would next walk to the target, whereas half anticipated that they would next throw to it. Although both groups had the same prior experience throwing the heavy ball, when making distance judgments, participants who were expecting to throw reported targets to be farther away than participants who were intending to walk.

\subsection{Method}

5.1.1 Participants. Twenty-four University of Virginia students (twelve male, twelve female) participated. Participants were either paid or recruited as part of a requirement for an introductory psychology course. All had normal or corrected-to-normal vision. They were naïve to the purpose of the experiment and had not participated in prior distanceperception experiments.

5.1.2 Apparatus and stimuli. The setup was the same as in experiment 1. Only the heavy ball was used. 
5.1.3 Design. Participants were assigned to either the intend-to-walk or the intend-tothrow condition. Six male and six female participants were in each condition. Each participant completed 1 practice block and 1 test block with 4 distances in each.

5.1.4 Procedure. Participants threw the heavy ball for all of the trials. They completed 4 practice distances and 4 test distances. The practice targets were placed at 3, 5, 9, and $11 \mathrm{~m}$, and the test targets were placed at $4,6,8$, and $10 \mathrm{~m}$. The orders of practice distances and test distances were randomized.

Participants threw the ball to each target three times. Participants were instructed to throw underhand with their dominant hand. After the third throw, participants estimated the distance to the targets. Half the participants were given the ball and asked to close their eyes and throw to the target. The other half of the participants was asked to put on a blindfold and to walk to the target. Participants were allowed to see their final performance. Those who walked to the target took off the blindfold and walked back to the starting point.

\subsection{Results and discussion}

A $2 \times 2 \times 4$ ANOVA with repeated measures on the last factor was run in order to determine whether intention influenced perceived distance. The independent factors were gender, intention, and distance. The dependent factor was estimated distance.

There was a main effect of intention condition $\left(F_{1,20}=4.787, p<0.05\right)$. Participants who were intending to blind throw estimated distances to be farther away than participants who were intending to blindwalk (see figure 5). The only other significant effect was for distance $\left(F_{3,60}=63.226, p<0.001\right)$.

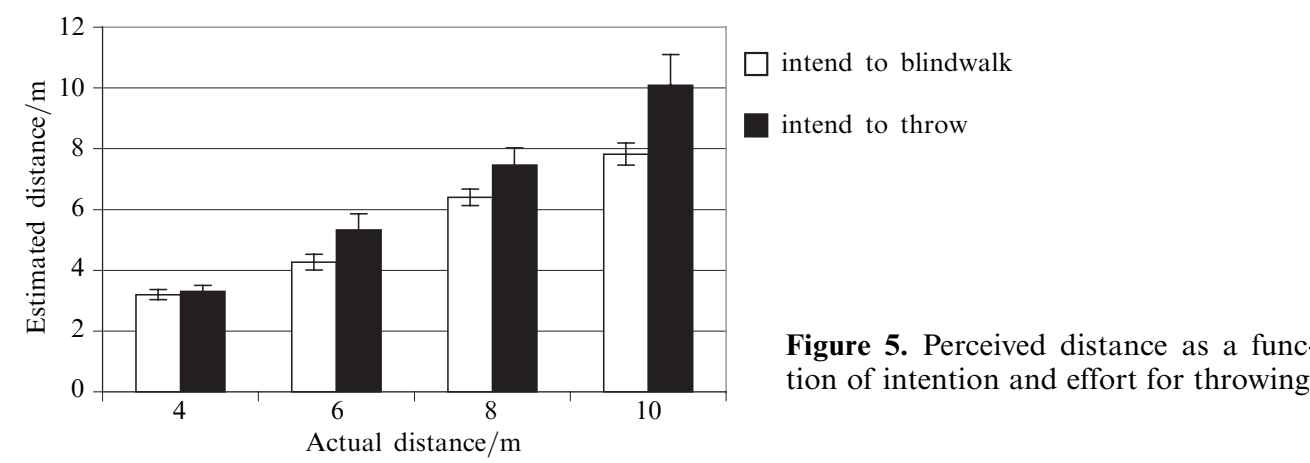

When participants were intending to throw, their conscious reports of distance were influenced by their prior experience throwing the heavy ball. However, when participants were intending to walk, throwing effort was irrelevant to the anticipated walking task, and therefore perceived distance did not increase. These results suggest that the effect of effort for throwing on conscious distance perception is functionally specific and depends on the action the perceiver intends to make.

\section{Experiment 5. Perceived distance: effort for walking and intention}

The previous experiment showed that effort for throwing influenced distance perception only when the perceiver was intending to throw and not when the perceiver was intending to walk. In this experiment, we manipulated anticipated walking effort by having participants walk on a treadmill while experiencing zero optic flow. Proffitt et al (2003) found that this manipulation caused a recalibration between walking energy and resulting optic flow, and thereby increased effort for walking, which induced an increase in apparent egocentric distance. In addition to having participants walk on a treadmill, we also manipulated their intentions by having them either blindwalk or throw to targets 
immediately after making their distance estimates. As with the previous experiment, this intention manipulation was defined by the action that participants anticipate performing next, either walking or throwing. We predicted that participants who anticipated walking would perceive targets as being farther away, as was found in the zero-optic-flow condition in experiment 3 by Proffitt et al. On the other hand, we predicted that participants who anticipated throwing would perceive the targets to be the same distance or possibly even closer after walking on the treadmill. (Proffitt et al found a decrease in apparent distance for participants who walked on the treadmill with canonical optic flow. It was felt that this decrease was due to the aerobic potentiation induced by the short brisk walk on the treadmill.) As predicted, we found that the treadmill-walking manipulation evoked an increase in apparent distance only for those participants who were intending to walk. Those who were intending to throw perceived targets to be nearer.

\subsection{Method}

6.1.1 Participants. Twenty-four University of Virginia students (twelve male, twelve female) participated. Participants were either paid or recruited as part of a requirement for an introductory psychology course. All had normal or corrected-to-normal vision. They were naïve to the purpose of the experiment and had not participated in prior distance-perception experiments.

6.1.2 Apparatus and stimuli. Distances were marked with a small orange disc cone in a hallway. Red construction paper covered the floor so participants could not count floor tiles as a way to estimate distance. For the practice distances, the cone was placed at 3, 5, 9, or $11 \mathrm{~m}$. Participants faced the same direction in the hall for all practice distances. Participants stood in a different place for each practice distance separated $1 \mathrm{~m}$ apart as a way to reduce the use of landmark cues as a reference for distance. The pre-adaptation phase was completed in a different section of the hallway. For the pre-adaptation distances, the cone was placed at 6, 8, and $10 \mathrm{~m}$, and the participants made their estimates starting from the same place. The post-adaptation distance was at $8 \mathrm{~m}$ at the same starting point as the pre-adaptation distances but in the opposite direction. Direction of pretest and posttest distances was counterbalanced.

6.1.3 Design. A motorized treadmill (Precor 9.1) was used. Half the participants were in the intend-to-walk condition. A small blindfold was used for these participants. The other half of the participants was in the intend-to-throw condition. These participants threw a small hacky-sack (a type of beanbag) filled with tiny beads.

We used a similar intention manipulation as in the previous experiment where half the participants performed one action after estimating the target distance, while the other half performed another action. In one condition, judgments were followed by blindwalking to the target; whereas in the other condition, participants threw the hacky-sack to the target after making each judgment. Six male and six female participants were in each condition. Each participant completed 1 practice block with 4 distances, 1 pretest block with 3 distances, and 1 posttest block with 1 distance. The presentation order of distances in each block was randomized.

6.1.4 Procedure. For the practice block, participants stood at one of four predetermined starting points and estimated the distance to each practice target. After making their estimates, half of the participants put on a blindfold and walked to the target, while the other half were given a hacky-sack and threw to the target. Both groups got visual feedback on their performance. After practice, participants were led to a different part of the hallway to complete the pre-adaptation block. The procedure was the same as for practice where participants estimated the distance and then would throw 
or blindwalk. Then participants walked on a treadmill at 3 miles $\mathrm{h}^{-1}$ for $3 \mathrm{~min}$. Note that 3 miles $^{-1}$ is a fairly typical walking speed and not one that would induce fatigue in $3 \mathrm{~min}$. At the end of $3 \mathrm{~min}$, a blindfold was placed over the participant's eyes, and the treadmill was stopped. Participants were led back into the hallway blindfolded and faced in the opposite direction as the pre-adaptation block. A target was placed at $8 \mathrm{~m}$ away. Participants removed the blindfold, estimated the distance to the target, and then either blindwalked or threw to the target.

\subsection{Results and discussion}

Ratios of posttest/pretest distance estimates for $8 \mathrm{~m}$ were calculated and used as the dependent measure in a 2 (gender) $\times 2$ (intention condition: throw or blindwalk) ANOVA. The main effect for intention condition was significant $\left(F_{1,20}=6.194, p<0.05\right)$. Relative to the pretests, participants in the blindwalk condition overestimated the target in the posttest condition while participants in the throw condition underestimated the target in the posttest condition (see figure 6). The underestimation in the throw condition is similar to the underestimation found by Proffitt et al (2003) in their canonicaloptic-flow condition. We hypothesize that the underestimation is due to a general muscle potentiation caused by the $3 \mathrm{~min}$ of brisk walking. Note that such a potentiation would affect both groups equally, and thus it is the difference between the two groups' judgments that reflects the magnitude of the treadmill manipulation for those participants who were intending to walk. No other effects were significant. These results suggest that perception is influenced by the effort associated with an intended action, and thus is functionally specific.

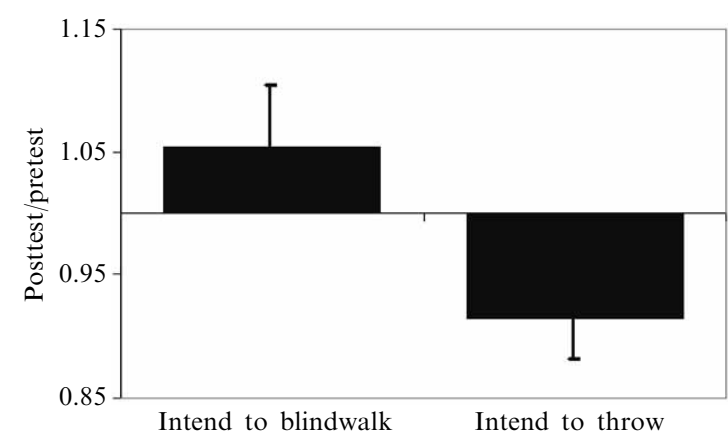

Figure 6. Perceived distance as a function of intention and effort for walking.

\section{General discussion}

Our findings indicate that the process underlying perception of egocentric distance combines the optically specified geometry of spatial layout with current behavioral goals and the potential to achieve these goals. Perceived distance is not only a function of the optical variables to which it relates, but it is also influenced by the actions we intend to perform and the effort associated with those actions.

Previous research has shown that effort for walking influences perceived geographical slant (Bhalla and Proffitt 1999; Proffitt et al 1995) and perceived distance (Proffitt et al 2003). The current research extends these findings in two ways. First, we showed that the influence of effort on perception is not limited to walking effort. In the first two experiments we showed that effort for throwing also influences conscious perceptions of distance. Second, we showed that the influence of effort on perception is functionally specific. In the third experiment we showed that the effort for throwing did not influence blindwalking, an action assessment of perceived distance that is functionally distinct from throwing. Moreover, experiments 4 and 5 showed that only the effort related to an intended action influences conscious distance perception. 
The influence of effort on perceived distance is conditioned by the specific action that we intend to perform. Existing models of perception do not take into account internal factors such as behavior potential and intention, and thus are in need of some revision.

According to Philbeck and Loomis (1997), the same perceptual information is used for both verbal judgments and blindwalking. As shown in figure $7 \mathrm{a}$, which is adapted from Philbeck and Loomis's figure 1, distance perception is generated by the processing of optical information. Different output transformations are applied to this information depending upon whether it is to be used for blindwalking or for conscious reports, but the value of perceived distance is the same regardless of the behavioral response. Philbeck and Loomis manipulated the type and number of depth cues, and found that both blindwalking and verbal reports were affected in a highly correlated manner as shown by the linear relationship found between the two responses. According to Philbeck and Loomis's model, in our experiment 3 blindwalking should have been influenced by the throwing manipulation as were conscious judgments in experiments 1 and 2. Instead, we found that throwing effort influenced verbal reports and visual matching but not blindwalking.

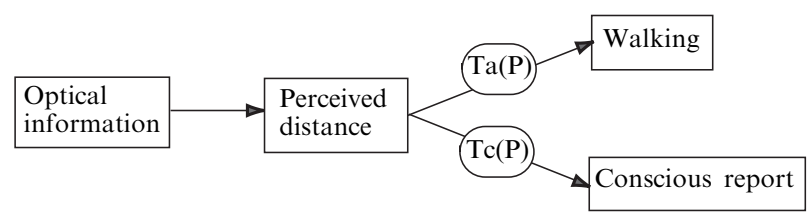

(a)

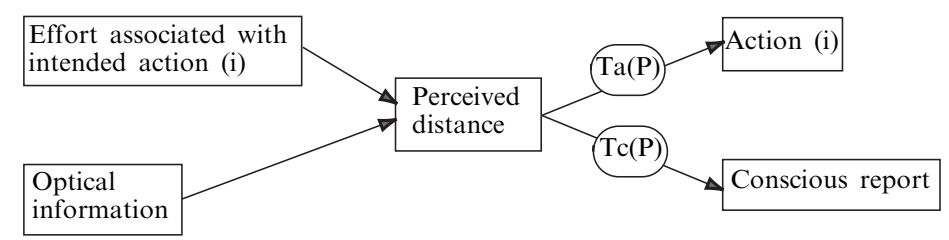

(b)

Figure 7. Different models of perception: (a) is adapted from Philbeck and Loomis (1997, figure 1). Optical information is processed and generates a perception. Then different output transformations are applied for blindwalking (Ta) and verbal estimates (Tc). (b) is a revision of Philbeck and Loomis's model. This new model takes into account what the perceiver is intending to do (action 'i').

Our results are consistent with Rieser et al's (1995) studies of visuomotor adaptation, which also demonstrated that a change in effort for throwing did not influence blindwalking. To manipulate effort for throwing, Rieser et al had participants throw to targets while standing on a moving trailer so that targets were either moving closer to them or farther away. After calibrating to throwing to targets moving towards them, participants threw too short when blind throwing to stationary targets. After calibrating to throwing to targets moving away from them, participants overthrew targets while blindfolded. However, the different throwing conditions did not affect blindwalking to targets. Table 1 shows how various manipulations of effort, such as those in the studies by Rieser et al (1995), Proffitt et al (2003), and the present studies, influence perceptual and action responses. 
Table 1. The effects of various manipulations of effort on conscious distance perception, blindwalking, and blind throwing.

\begin{tabular}{|c|c|c|c|}
\hline \multirow{2}{*}{ Manipulation } & \multicolumn{3}{|l|}{ Distance Response } \\
\hline & conscious perception & blindwalking & blind throwing \\
\hline Effort for walking & affected $^{(a)}$ & affected $^{(a),(b)}$ & unaffected $^{(b)}$ \\
\hline Effort for throwing & affected $^{(\mathrm{c})}$ & unaffected $^{(b),(d)}$ & affected $^{(b)}$ \\
\hline
\end{tabular}

Our findings, that conscious judgments of distance were influenced by throwing effort but that blindwalking was not, demonstrate a dissociation between conscious reports of distance and blindwalking. This suggests a revision to Philbeck and Loomis's account. As shown in figure $7 \mathrm{~b}$, the perceptual representation is influenced by both optical information and the effort associated with intended actions. As the effort required for an intended action changes, perceived distance also changes. This account is compatible with results demonstrating a close relationship between perceptual reports and blindwalking (Philbeck and Loomis 1997). This account is also consistent with the functional specificity in Rieser et al's (1995) findings on visuomotor adaptation. We found that a change in effort for throwing will influence perception when the perceiver is intending to (and subsequently does) throw (experiments 1 and 2), but not when the perceiver is intending to (and subsequently does) walk (experiment 4). Likewise, a change in effort for walking only influences perception when the perceiver is intending to walk (experiment 5).

Our experiments 4 and 5 showed that it is the effort associated with an intended action that influences perception. In experiment 4, effort for throwing increased by having all participants throw a heavy ball. Half the participants made their verbal judgments with the intention to blind throw, while the other half intended to blindwalk. Only participants intending to blind throw were influenced by anticipated throwing effort. These participants perceived the distances to be farther away than participants intending to blindwalk despite both groups having experienced the same throwing adaptation. In experiment 5, effort for walking was increased by pairing forward walking energy with zero optic flow. In this study, only participants intending to blindwalk judged distances to be greater while participants intending to blind throw were unaffected by the recalibration. Perceived distance is influenced by the effort related to the intended action. If there is no change in the effort required to perform the intended action, then conscious distance perception will be unaffected despite possible changes to effort for other actions (see table 2). In both experiments, both groups of participants experienced the same experimental manipulation on effort. But the differences in the actions that the different groups were intending to perform after their verbal judgment altered their perception of distance. Perception informs us about how the geometry of spatial layout relates to our intentions and behavior potential.

Table 2. The effect of changes in effort for particular actions on conscious distance perception after adaptation given the perceiver's intention.

\begin{tabular}{lll}
\hline Intention & Adaptation & \\
\cline { 2 - 3 } & throwing (experiment 4) & walking (experiment 5) \\
\hline To throw & change & $\begin{array}{l}\text { no change } \\
\text { change }\end{array}$
\end{tabular}


This account of perception makes two very general claims. First, perception is at the same time about the environment and about the self. The spatial layout of the environment is perceived relative to the actions that it affords and the effort associated with those actions that we intend to pursue. Second, this account is a functional one, grounded in the pragmatic philosophy of James (1890) and its development by Gibson (1979). [See Heft (2001) for the relationship between the approaches of James and Gibson.] Perception relates the geometry of the environment, as specified by optical variables, to the goals and behavioral capabilities of the perceiver.

\section{Conclusions}

Perceived distance is a function of (i) actual distance as specified by optical variables, (ii) what we are intending to do, and (iii) the effort associated with this intended action. Phenomenal distance is a measure of the effort required to perform distancerelative actions. If our intent is to walk to a target, then we see distance in terms of the effort required to walk to it. Similarly, if our intent is to throw a ball to a target, then distance is perceived in terms of throwing effort. We see the world in terms of the potential actions that it affords and the effort associated with these actions.

Acknowledgments. This research was supported by NSF ITR/Carnegie Mellon Grant 0121629, and ONR Grant N000140110060, to the second author.

\section{References}

Amorim M-A, Loomis J M, Fukusima S S, 1998 "Reproduction of object shape is more accurate without the continued availability of visual information" Perception $2769-86$

Anstis S, 1995 "Aftereffects from jogging" Experimental Brain Research 103 476-478

Bhalla M, Proffitt D, 1999 "Visual-motor recalibration in geographical slant perception" Journal of Experimental Psychology: Human Perception and Performance 25 1076-1096

Durgin F H, Pelah A, 1999 "Visuomotor adaptation without vision" Experimental Brain Research $12712-18$

Epstein W, Rogers S J, 1995 Perception of Space and Motion. Handbook of Perception and Cognition 2nd edition (Portland, OR: Academic Press)

Gibson J J, 1979 The Ecological Approach to Visual Perception (Boston, MA: Houghton Mifflin)

Heft H, 2001 Ecological Psychology in Context: James Gibson, Roger Barker, and the Legacy of William James's Radical Empiricism (Mahwah, NJ: Lawrence Erlbaum Associates)

James W, 1890 The Principles of Psychology (New York: Holt)

Loomis J M, Da Silva J A, Fujita N, Fukusima S S, 1992 "Visual space perception and visually directed action" Journal of Experimental Psychology: Human Perception and Performance 18 $906-921$

Norman J F, Todd J T, Perotti V J, Tittle J S, 1996 "The visual perception of three-dimensional length" Journal of Experimental Psychology: Human Perception and Performance 22 173-186

Philbeck J W, Loomis J M, 1997 "Comparison of two indicators of perceived egocentric distance under full-cue and reduced-cue conditions" Journal of Experimental Psychology: Human Perception and Performance $\mathbf{2 3} 72-85$

Proffitt D R, Bhalla M, Gossweiler R, Midgett J, 1995 "Perceiving geographical slant" Psychonomic Bulletin \& Review 2 409-428

Proffitt D R, Caudek C, 2002 "Depth perception and perception of events", in Handbook of Psychology Ed. I B Weiner, volume 4 Experimental Psychology Eds A F Healy, R W Proctor (New York: Wiley) pp $213-216$

Proffitt D, Stefanucci J, Banton T, Epstein W, 2003 "The role of effort in distance perception" Psychological Science $14106-113$

Rieser J J, Pick H L, Asmead D H, Garing A E, 1995 "Calibration of human locomotion and models of perceptual-motor organization" Journal of Experimental Psychology: Human Perception and Performance $21480-497$ 


\section{PERCEPTION}

vOLUME 332004

www.perceptionweb.com

Conditions of use. This article may be downloaded from the Perception website for personal research by members of subscribing organisations. Authors are entitled to distribute their own article (in printed form or by e-mail) to up to 50 people. This PDF may not be placed on any website (or other online distribution system) without permission of the publisher. 